\title{
Aggressive angiomyxoma pelvis presenting as an ovarian tumor
}

\author{
Errarhay Sanaa $^{1 *}$, Lamquami Safae $^{1}$, Mahmoud Samia $^{1}$, El Fatemi Hinde $^{2}$, Saadi Hanane ${ }^{1}$, \\ Squalli Nadia $^{3}$, Bouchikhi Chahrazed ${ }^{1}$, Amarti Afaf ${ }^{2}$, Banani Abdelaziz ${ }^{1}$ \\ ${ }^{1}$ Department of Gynecology and Obstetrics, University Hospital of Fez, Fez, Morocco \\ ${ }^{2}$ Department of Pathology, University Hospital of Fez, Fez, Morocco \\ ${ }^{3}$ Department of Radiology, University Hospital of Fez, Fez, Morocco \\ Email: suine_err@yahoo.fr
}

Received 20 August 2013; revised 18 September 2013; accepted 26 September 2013

Copyright (c) 2013 Errarhay Sanaa et al. This is an open access article distributed under the Creative Commons Attribution License, which permits unrestricted use, distribution, and reproduction in any medium, provided the original work is properly cited.

\begin{abstract}
Aggressive angiomyxoma is rare mesenchymal tumor that most commonly arises in the vulvo-vaginal region, perineum, and pelvis of women. The term aggressively emphasizes the often infiltrative nature of the tumor and its frequent association with local recur- rence. Preoperative diagnosis and treatment may be difficult. We report a 55 -year-old patient who presented with a pelvic mass with no significant medical history. The radiological assessment showed a pelvic cystic tumor retro and lateral bladder. A surgical excision was performed with a diagnosis of aggressive angiomyxoma pelvis that misdiagnosed as ovarian tumor. The patient received adjuvant radiotherapy after surgery and remains well with no signs of local recurrence at 12 months follow-up. As surgery is the main treatment, preoperative diagnosis and assessment of extent of the tumor for planning surgery can not be overemphasized. The authors discuss this finding through the diagnostic difficulties and the evolution of this entity.
\end{abstract}

Keywords: Aggressive Angiomyxoma; Pelvic Neoplasm; Immunohistochemistry

\section{INTRODUCTION}

Aggressive angiomyxoma is rare mesenchymal tumor that most commonly arises in the vulvo-vaginal region, perineum, and pelvis of women. The term aggressively emphasizes the often infiltrative nature of the tumor and its frequent association with local recurrence. Preoperative diagnosis and treatment may be difficult.

\footnotetext{
*Corresponding author.
}

\section{CASE REPORT}

A 55-year-old woman, followed for diabetes under treatment, she had a surgical history of hysterectomy, the histopathology showed a uterine leiomyoma with adenomyosis. The patient presented in our department with progressive distension of abdomen, there was not history of loss of appetite or weight lost. The vaginal speculum showed a macroscopically healthy, the bimanual vaginal examination revealed distended abdomen non tender, with diffuse, firm, painless and ill-defined mass (Figures 1-6).

On ultrasonography, we found two complexes and solids lateral and retro bladder masses, avascular with Doppler, each one measured $100 \times 80$ and $30 \mathrm{~mm}$, with mild bilateral hydro-ureteronephrosis.

Pelvic MRI showed a retro bladder mass $90 \times 90 \times 90$ $\mathrm{mm}$ that enhanced well and heterogeneous with gadolinium, it's include hypo intensity onT1-weighted images and hyper intensity on T2-weighted images . MRI indicated that the mass did not involve the rectal wall or bladder. The mass did not appear to involve pelvic musculature but involvement of the proximal vaginal wall could not be ruled out.

An exploratory laparotomy was done and the tumor was excised incompletely, the tumor was found to be bilobed and unencapsulated, measuring approximately $150 \mathrm{~mm}$. the liver was normal and there was no ascites or carcinomatosis. A complete surgical resection was impossible without risk of neurovascular and urologic sequels; the mass was partially removed in multiple fragments.

Aggressive angiomyxoma was finally diagnosed by pathologists, the tumor was infiltrative, embedded in a myxoid stroma with an important vascular component and the tumor cells were immune-reactive for Desmin 


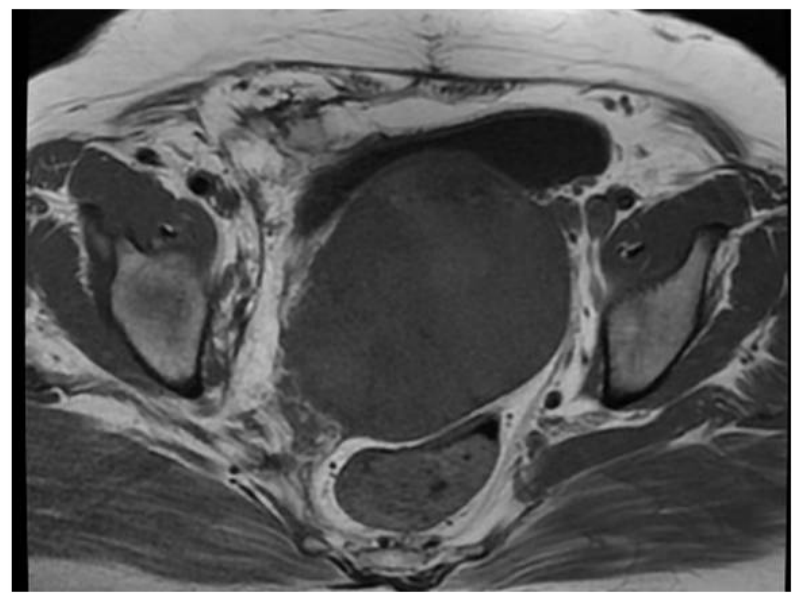

Figure 1. T1 Weighted MR image: homogeneous isointense retro bladder mass.

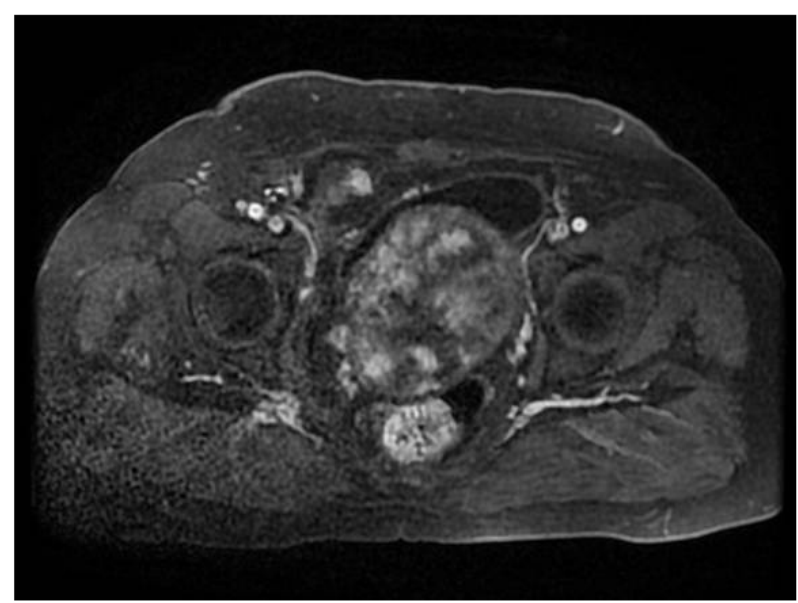

Figure 2. The mass has heterogeneous increased signal intensity at T2-weighted MR imaging and is heterogeneously enhanced after intravenous administration of gadodiamide.

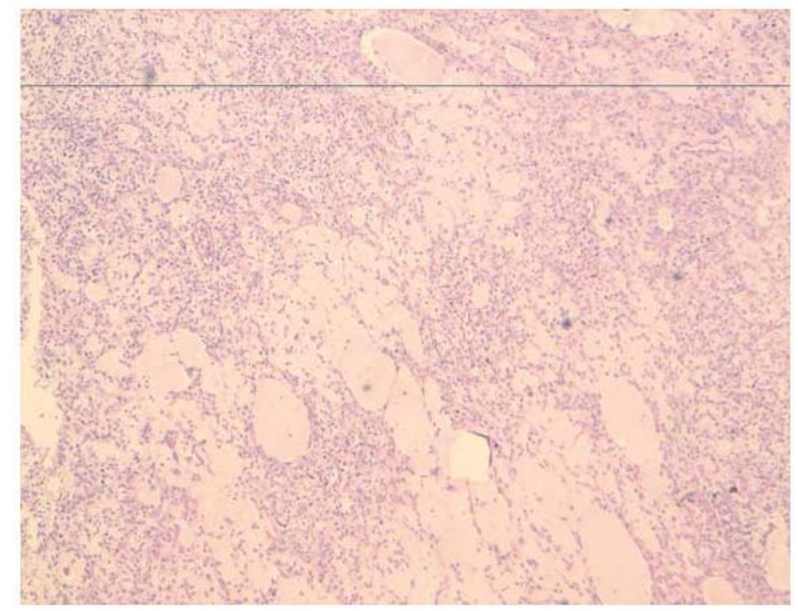

Figure 3. HES X 4: agressive angiomyxoma. Prominent thinand thick-walled vascular channels surrounded by a myxoid stroma.

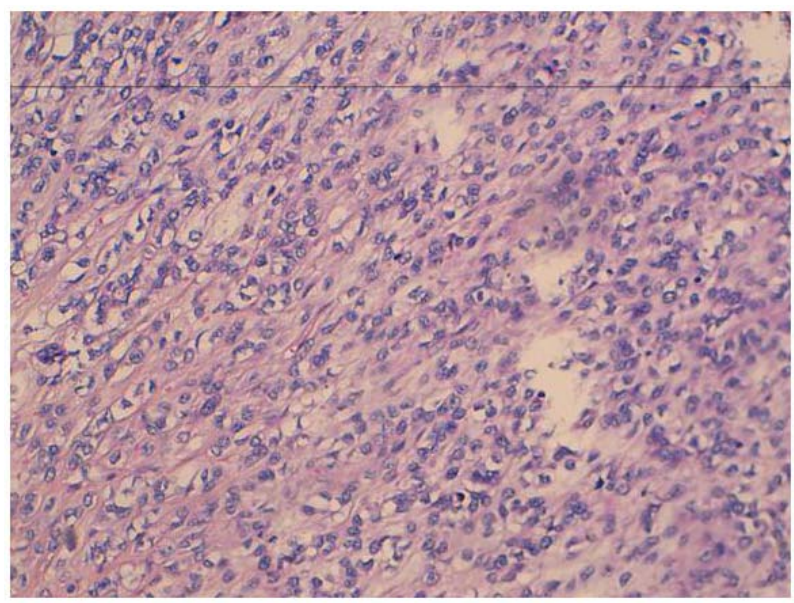

Figure 4. HES $\times 10$ : bland spindled and stellate-shaped cells.

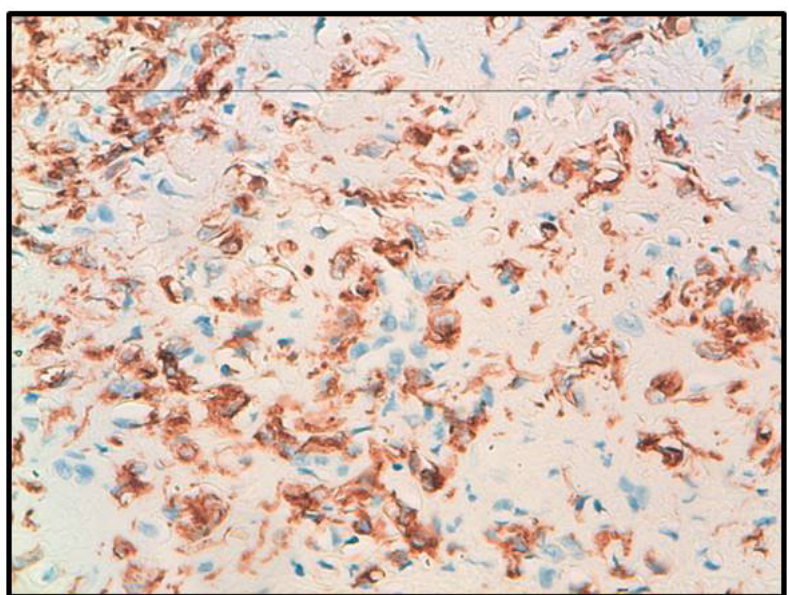

Figure 5. Diffuse desmin immunoreactivity in an aggressive angiomyxoma.

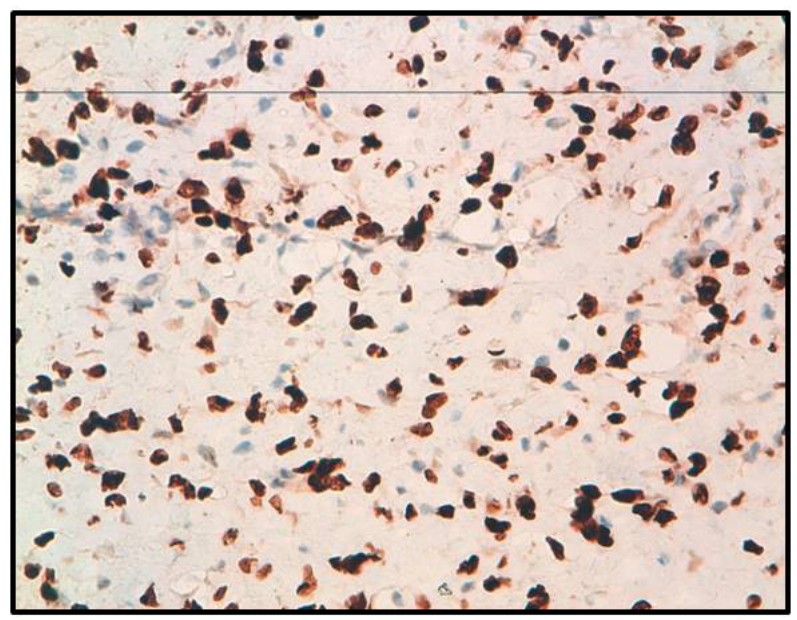

Figure 6. Diffuse estrogen receptor immunoreactivity in an aggressive angiomyxoma.

and receptors hormones, immune-negative for other immune-reactive (anti AML, anti PS 100, anti CK, anti CD 
117)

Postoperatively, the pelvic examination has objectified a small and firm vaginal mass, CT scan was done after two months showed residual pelvic tumor inter rectumbladder $50 \times 30 \mathrm{~mm}$ and the patient have extensive surgery with partial right urethral resection with ureteral reimplantation at the bladder and she received adjuvant radiotherapy and remains well with no signs of local recurrence at 12 months follow-up.

\section{DISCUSSION}

A wide variety of mesenchymal lesions occur in the lower female genital tract [1,2]. Broadly, these mesenchymal lesions can be separated into two groups. The first group includes several well-characterized tumors that show a marked tendency to occur in the lower female genital tract, such as aggressive angiomyxoma, angiomyofibroblastoma and cellular angiofibroma [3,4].

These tumors can also be called as relatively site-specific. The second group embraces a wide range of heterogeneous lesions that frequently occur in this region, but arise in other anatomic sites as well, with examples as fibroepithelial stromal polyp and superficial angiomyxoma.

Agrressive Angiomyxoma (AAM) is a rare mesenchymal tumor arising from the soft tissues of pelvis or perineum. It occurs mainly in the reproductive age group [5]. The size may vary from $1-60 \mathrm{~cm}$. The diagnosis can be made considering the clinical presentation aided by ultrasound, Computerized tomography scan (CT) or MRI showing a hypodense mass with translevator extension, displacing rather than invading the pelvic organs [6].

Histopathology alone gives the definite diagnosis. It has low cellularity with spindle shaped cells in a loose matrix containing medium sized arteries having fibrointimal proliferation. Most tumors have been found to have estrogen and progesterone receptor positive status. Tumor cells are atleast focally immune-reactive to desmin, smooth muscle actin, muscle specific actin and vimentine [6].

Surgery is the mainstay of treatment [5]. Radiotherapy and chemotherapy may not have much role due to the low mitotic activity seen [7] GnRH agonist [8] and tamoxifen have been used successful in few patients.

The first line of the therapy for AAM is surgery, although achieving negative resection margins are difficult because of the infiltrative nature of the tumor and the absence of a defined capsule. Smaller, more-superficial tumors of the vulva or vagina may be removed with wide, local excision, but larger, deep-seated tumors of the pelvis may require more extensive surgery with partial or complete resection of some pelvic organs, conferring a higher risk of morbidity. In the current study, a large sur- gery was performed. Even though complete surgical resection is the desired goal, incomplete removal is acceptable when significant operative morbidity is anticipated or when preservation of fertility is a concern [5].

Chemotherapy and radiation therapy do not have welldefined roles in the treatment of AAM. Preoperative and intraoperative radiations have been used, together with tumor embolization, to decrease the risk of recurrence in a patient with a large pelvic AAM, but no follow-up data were provided.

Radiation therapy has also been used to treat several cases of tumor recurrence reported tumor-free intervals of 2 to 3 years $[9,10]$.

A newer approach to the treatment of AAM involves hormonal therapy. Given that the tumor occurs primarily in premenopausal women and is positive for estrogen receptor and progesterone receptor, estrogen and progesterone may play a role in its development. Several case reports using a gonadotropin-releasing hormone agonist as medical management for AAM showed complete radiographic resolution of the tumor $[11,12]$.

Although surgery remains the standard of care, the medical treatment of AAM with a gonadotropin-releaseing hormone agonist in the primary or adjuvant setting may offer an alternative to radical surgery [11].

Despite the morbidity associated with tumor recurrence and repeat surgeries, the prognosis for patients with AAM is generally considered good. Recurrence rates from the largest case series, [5] range from 25\% to $47 \%$, with $85 \%$ of those occurring within 5 years of initial surgery. [5] Close clinical follow-up with imaging studies has been advocated.

\section{CONCLUSION}

Aggressive Angiomyxoma (AAM) is a rare mesenchymal tumor arising from the soft tissues of pelvis or perineum. Surgery is the mainstay of treatment. Radiotherapy and chemotherapy may not have important role due to the low mitotic activity. GnRH agonist and tamoxifen have been used successfully in few patients. The prognosis for patients with AAM is generally considered good.

\section{REFERENCES}

[1] McCluggage, W.G. (2005) A review and update of morphologically bland vulvovaginal mesenchymal lesions. International Journal of Gynecologic Pathology, 24, 2638.

[2] Nucci, M.R. and Fletcher, C.D. (2000) Vulvovaginal soft tissue tumors: Update and review. Histopathology, 36, 97-108.

http://dx.doi.org/10.1046/j.1365-2559.2000.00865.x

[3] Steeper, T.A. and Rosai, J. (1983) Aggressive angiomyxoma of the female pelvis and perineum: Report of nine 
cases of a distinctive type of gynecologic soft-tissue neoplasm. American Journal of Surgical Pathology, 7 463475. http://dx.doi.org/10.1097/00000478-198307000-00009

[4] Nucci, M.R., Granter, S.R. and Fletcher, C.D. (1997) Cellular angiofibroma: A benign neoplasm distinct from aggressive angiomyxoma. American Journal of Surgical Pathology, 21, 636-644. http://dx.doi.org/10.1097/00000478-199706000-00002

[5] Chan, Y.M., Hon, E., Ngai, S.W., Ng, T.Y. and Wong, L.C. (2000) Aggressive angiomyxoma in females: Is radical resection the only option? Acta Obstetricia et Gynecologica Scandinavica, 79, 216-220. http://dx.doi.org/10.1080/j.1600-0412.2000.079003216.x

[6] Fetsch, J.F., Laskin, W.B., Lefkowitz, M., Kindblom, L.G. and Meis-kindblom, J.M. (1996) Aggressive angiomy- xoma: A clinicopathologic study of 29 female patients. Cancer, 78, 79-90.

http://dx.doi.org/10.1002/(SICI)1097-0142(19960701)78: 1<79::AID-CNCR13>3.0.CO;2-4

[7] Bigby, S.M., Symmans, P.J., Miller, M.V., Drays, M.S. and Jones, R.W. (2011) Aggressive angiomyxoma of the female genital tract and pelvis clinicopathologic features with immunohistochemical analysis. International Journal of Gynecologic Pathology, 30, 505-513.

\section{http://dx.doi.org/10.1097/PGP.0b013e318211d56c}

[8] Behranwaha, K.A. and Thomas, J.M. (2003) Aggressive angiomyxoma: A distinct clinical entity. European Journal of Surgical Oncology, 29, 559-563. http://dx.doi.org/10.1016/S0748-7983(03)00104-5

[9] Rhombrg, W., Jasarevic, Z., Alton, R., Kompatscher, P., Beer, G. and Breitfellner, G. (2000) Aggressive angiomyxoma: Irradiation for recurrent disease. Strahlentherapie und Onkologie, 176, 324-326. http://dx.doi.org/10.1007/s000660050015

[10] Suleiman, M., Duc, C., Ritz, S. and Beieri, S. (2006) Pelvic excision of large aggressive angiomyxoma in a woman: Irradiation for recurrent disease. International Journal of Gynecological Cancer, 16, 356-360. http://dx.doi.org/10.1111/j.1525-1438.2006.00218.x

[11] Fine, B.A., Munoz, A.K., Ltz, C.E. and Gershenson, D.M. (2001) Primary medical management of recurrent aggressive angiomyxoma of the vulva with a gonadotropin-releasing hormone agonist. Gynecologic Oncology, 81, 120122. http://dx.doi.org/10.1006/gyno.2000.6119

[12] McCluggage, W.G., Jamieson, T., Dobbs, S.P. and Grey, A. (2006) Aggressive angiomyxoma of the vulva: Dramatic response to gonadotropin-releasing hormone agonist therapy. Gynecologic Oncology, 100, 623-625. http://dx.doi.org/10.1016/j.ygyno.2005.09.033 